\title{
Regret and fear in prostate cancer: The relationship between treatment appraisals and fear of recurrence in prostate cancer survivors
}

\author{
Rebecca Maguire $^{1}$ (D) | Paul Hanly ${ }^{1}$ | Frances J. Drummond ${ }^{2}$ | Anna Gavin ${ }^{3}$ | Linda Sharp ${ }^{4}$
}

${ }^{1}$ National College of Ireland, Dublin, Ireland

${ }^{2}$ University College Cork, Cork, Ireland

${ }^{3}$ Queen's University Belfast, Cork, Ireland

${ }^{4}$ Newcastle University, Newcastle upon Tyne, United Kingdom

Correspondence

Rebecca Maguire, National College of Ireland, Mayor Street, Dublin 1, Ireland.

Email: rebecca.maguire@ncirl.ie

\begin{abstract}
Objective Fear of recurrence (FOR) is a key concern among survivors of all cancers. In prostate cancer, FOR varies with health and treatment type, but little is known about how survivors' appraisals of their treatment, and in particular, their level of regret over treatment decisions may affect this.

Methods A total of 1229 prostate cancer survivors between 2- and 5-years postdiagnosis were invited to complete a postal questionnaire including a FOR scale, Decisional Regret Scale, and the European Organization for Research and Treatment of Cancer QLQ C30 health-related quality of life (HRQoL) instrument. Multiple regression analysis explored the impact of 3 blocks of factors on FOR: (1) demographic characteristics and disease extent, (2) primary treatment received and health status (treatment side effects and HRQoL), and (3) treatment appraisals, specifically satisfaction with information received regarding treatment and level of regret experienced over treatment decisions.
\end{abstract}

Results The final multivariable model explained $27 \%$ of variance on FOR. Significant correlates of lower FOR included having localised disease, having undergone an invasive treatment, as well as health status (higher HRQoL and fewer ongoing side effects). Beyond this, appraisals of treatment significantly contributed to the model: More decisional regret and lower satisfaction with information received were associated with higher FOR.

Conclusion These findings suggest that FOR may be mitigated by providing survivors with more information regarding treatment choices and the treatment itself so that men can make well-informed decisions and experience less future regret.

Sensitivity analysis for variables predicting FOR among prostate cancer survivors is not suspected of having a recurrence.

\section{KEYWORDS}

appraisals, cancer, fear of recurrence, Ireland, oncology, prostate cancer, regret, treatment decision making

\section{BACKGROUND}

In recent years, the incidence of prostate cancer (PC) has been increasing internationally, ${ }^{1}$ with $\mathrm{PC}$, the most common invasive cancer among men, accounting for approximately $40 \%$ of male cancer survivors in developed countries. ${ }^{2}$ While this increased incidence may be due to the upsurge in prostate specific antigen testing, recent research suggests such testing has no effect on mortality rates. ${ }^{3}$ However, the range of treatment options available, including radical prostatectomy (RP), external beam radiotherapy (ERBT), brachytherapy (BT), and androgen deprivation therapy (ADT), has contributed to 5-year survival rates of nearly $100 \% .{ }^{4}$ Yet, despite this good prognosis, a diagnosis of PC can be accompanied by numerous adverse psychological effects, including anxiety and depression. ${ }^{5}$ These feelings are often exacerbated by-or even attributed to-worry over cancer, and in particular, a fear that the cancer will return or progress. ${ }^{6}$ Typically referred to as 
"fear of recurrence" (FOR), this experience is common within PC survivors, ${ }^{7,8}$ second only to concerns over sexual dysfunction. ${ }^{9}$

Given the detrimental effect that FOR can have on various aspects of well-being, ${ }^{10}$ the importance of recognising and seeking to treat this is widely acknowledged. ${ }^{11}$ In PC, the negative impact of this worry is evident, with higher FOR associated with lower quality of life (QOL), higher distress, and more physical problems in survivors. ${ }^{8}$ Conversely, lower FOR has been shown to significantly correlate with better mental health scores. ${ }^{10}$ In addition, although FOR often improves after PC treatment, it does not disappear nor change substantially in the 2 years thereafter. ${ }^{7}$ While a handful of studies have begun to explore what exacerbates FOR in $\mathrm{PC},{ }^{7-9,12}$ these studies are often limited to survivors undergoing a particular treatment (eg, RP), have small samples, or focus on a limited range of sociodemographic and clinical variables. A more thorough analysis of potential correlates of FOR in $P C$ is therefore merited.

Understanding the role that cognitive appraisals play in the process may offer insights into steps that may be taken to mitigate FOR. ${ }^{12}$ One of the key influences on FOR may be survivors' experience and appraisals of their treatment; yet few studies have systematically investigated this as distinct from the treatment itself. While there has been a move for patients to have a more active role in their treatment choice, PC patients are often not well informed about treatments and their consequences. ${ }^{13} \mathrm{PC}$ treatments are often accompanied by significant and long-lasting adverse side effects including urinary incontinence, sexual dysfunction, bowel problems, fatigue, and insomnia. ${ }^{14,15}$ While survivors can adapt to such negative outcomes, ${ }^{16}$ these effects can have a detrimental impact on QOL, making it difficult to cope postdiagnosis. ${ }^{17}$ Any side effects experienced are likely to contribute to FOR, but this may be exacerbated further should patients feel ill-informed as to what to expect from their treatment. Moreover, although generally low in PC survivors, a significant minority experience considerable treatment regret, ${ }^{18-21}$ which could have the potential to influence FOR.

The aim of this study is to investigate the associations between PC survivors' treatment appraisals and FOR. Specifically, we hypothesise that lower satisfaction with information received regarding treatment and higher treatment regret will be associated with greater FOR after controlling for established correlates, including sociodemographic and clinical factors, treatment received, and health status.

\section{2 | METHODS}

\section{1 | Participants}

This study formed part of the PiCTure (prostate cancer treatment, your experience) study-a project involving PC survivors in the Republic of Ireland and Northern Ireland. ${ }^{22,23}$ This aspect of the study was limited to survivors in Republic of Ireland. Following ethical approval from the Irish College of General Practitioners, PC survivors at least 2-year postdiagnosis were identified from the population-based National Cancer Registry, Ireland. Following screening for eligibility by general practitioners, 4453 survivors were invited to complete a postal questionnaire between April and September 2012. In addition to a core questionnaire administered to all survivors, those who were less than 5 -year postdiagnosis $(N=1229)$ were asked to complete a FOR scale. We focused on this subset of participants in the current analysis.

\section{3 | INSTRUMENTS}

\subsection{Fear of recurrence}

Fear of recurrence was measured using a 5 -item scale, ${ }^{24,25}$ previously validated in PC populations. ${ }^{7,9}$ This requires survivors to report worries over cancer ("I will probably relapse within the next 5 years", "My fear of cancer getting worse gets in the way of my enjoying life", "Because cancer is unpredictable, I feel I cannot plan for my future", and "I am afraid of my cancer getting worse") as well as one item relating to optimism over prognosis ("I am certain that I have been cured of cancer"). Items are scored on a 5-point Likert scale with total values standardised to provide a score of 0 to 100 (higher scores correspond to greater FOR). Reliability for this measure was good in our sample with a Cronbach a of .84.

\subsection{Sociodemographic and clinical information}

Participants provided sociodemographic information including age, marital status, employment status, and highest level of education. Respondents were classified by disease extent (localised vs locally advanced/advanced) at time of diagnosis on the basis of clinical stage and Gleason Grade (GG) as obtained from National Cancer Research Institute records. Survivors with stage I/II disease and a GG of 2 to 7 at diagnosis were considered to have localised disease, whereas those having stage 3 to 4 with any GG were classified as having locally advanced/advanced disease. Survivors having any other combinations of stage and GG were excluded.

\section{3 | Treatment and health status}

As described previously, ${ }^{22}$ survivors were classified according to primary treatment received, providing a single variable broadly capturing both the main treatment(s) and their invasiveness. Categories were RP, EBRT with concurrent ADT within 6 months, and EBRT without concurrent ADT, BT, and ADT alone. Those who had not been treated but had undergone observation/surveillance only (ie, watchful waiting and/or active surveillance) were also included.

Survivors indicated if they were currently experiencing any side effects from their treatment, including urinary incontinence, loss of sexual desire, bowel problems, breast swelling/tenderness, sweats or hot flashes, fatigue, and/or depression. ${ }^{26} \mathrm{~A}$ total number of ongoing side effects were computed.

The Global Health Score (GHS) subscale of the European Organization for Research and Treatment of Cancer ${ }^{27}$ QLQ30 was used as a general measure of health-related quality of life (HRQoL). This is derived from 2 items measuring general health ("How would you rate your overall physical condition during the past week?") and perceived QOL ("How would you rate your overall quality of life during the past week?"). Responses are measured on a 7-point Likert scale 
(from poor to excellent) and standardised into a score ${ }^{27}$ of 0 to 100 Higher GHS indicates better HRQoL.

\subsection{Appraisals of treatment: satisfaction with treatment information and decisional regret}

Survivors were asked to reflect on how satisfied they were with the amount of information given to them about their treatment overall using a single item rated on a scale of 1 (not at all satisfied) to 7 (extremely satisfied). The Decisional Regret Scale ${ }^{28}$ was used to measure survivor's regret. Here, participants were asked to reflect on the entire treatment experience by rating their agreement with 3 positively phrased ("It was the right decision", "I would go for the same choice if I had to do it over again", and "The decision was a wise one") and 2 negatively phrased ("I regret the choice that was made", and "The choice did me a lot of harm") statements on a scale of 1 (strongly agree) to 5 (strongly disagree). After positively phrased items are recoded, scores are summed and standardised to give rise to a value of 0 to 100 (higher scores reflect higher regret). This scale has good psychometric properties ${ }^{28}$ with a Cronbach $a$ in our sample of .85 .

TABLE 1 Descriptive statistics for prostate cancer survivors

\begin{tabular}{|c|c|c|c|c|}
\hline Categorical Variables & No. & $\%$ & & FOR \\
\hline \multicolumn{5}{|l|}{ Education } \\
\hline Primary & 225 & 27.5 & & 31.0 \\
\hline Third level or higher & 223 & 27.3 & & 27.6 \\
\hline Missing & 27 & 3.3 & & \\
\hline \multicolumn{5}{|l|}{ Marital status } \\
\hline Married/cohabiting & 658 & 80.5 & & 28.7 \\
\hline Other & 151 & 18.5 & & 32.8 \\
\hline Missing & 8 & 1.0 & & \\
\hline Total & 817 & 100 & & \\
\hline \multicolumn{5}{|l|}{ Employment status at diagnosis } \\
\hline Total & 817 & 100 & & \\
\hline \multicolumn{5}{|l|}{ Disease classification at diagnosis } \\
\hline Localised & 488 & 59.7 & & 26.9 \\
\hline Advanced & 130 & 15.9 & & 35.9 \\
\hline Missing & 199 & 24.4 & & \\
\hline Total & 817 & 100 & & \\
\hline \multicolumn{5}{|l|}{ Primary treatment } \\
\hline $\mathrm{RP}$ & 258 & 31.6 & & 25.5 \\
\hline ERBT with ADT & 156 & 19.1 & & 30.9 \\
\hline Continuous variables & Mean & SD & Range & Possible range \\
\hline Survivor age & 68.48 & 7.87 & $47-91$ & $18+$ \\
\hline Time since diagnosis (years) & 3.41 & 1.13 & $2-5$ & $2-5$ \\
\hline Global health score (GHS) & 71.61 & 21.15 & $0-100$ & $0-100$ \\
\hline Number of side effects & 2.16 & 1.72 & $0-8$ & $0-8$ \\
\hline Regret over decision & 18.02 & 19.56 & $0-100$ & $0-100$ \\
\hline Satisfaction with info received & 6.04 & 1.34 & $1-7$ & $1-7$ \\
\hline FOR & 29.42 & 20.97 & $0-100$ & $0-100$ \\
\hline
\end{tabular}

Abbreviations: ADT, androgen deprivation therapy; BT, brachytherapy; ERBT, external beam radiotherapy; FOR, fear of recurrence; RP, radical prostatectomy. 


\section{5 | Possible recurrence}

Clinical information on recurrence was not available. However, if survivors received chemotherapy or received ADT or ERBT more than 6 months after their primary treatment, they were classified as having evidence of a possible recurrence. ${ }^{22}$

\section{6 | Statistical analysis}

A hierarchical multiple regression model was developed to examine the impact of 3 blocks of factors on FOR: (1) sociodemographic characteristics (age, education, employment status, and marital status) and disease extent at diagnosis, (2) primary treatment type and health status (number of side effects and HRQoL), and (3) treatment appraisals (treatment regret, and satisfaction with treatment). Descriptive statistics were calculated including means, ranges, and standard deviations. No violations regarding assumptions of normality, linearity, and homoscedasticity were observed. Examination of correlations amongst independent variables revealed no problems with multicollinearity. Associations with FOR were assessed using 2-sided $t$ tests and $P$-values of $<.05$ considered significant.

A sensitivity analysis was performed where the model was rerun excluding survivors suspected of having a recurrence (excluded $n=222 ; 27 \%)$.

\section{4 | RESULTS}

\section{1 | Survivor characteristics}

Of the 1229 eligible survivors, 817 (response rate 66.5\%) completed the questionnaire. Table 1 displays frequency information and mean
FOR scores for the categorical variables and descriptive statistics for the continuous variables. The majority of survivors (79\%) had localised disease and had undergone some form of invasive treatment, with RP (32\%) and ERBT without concurrent ADT (32\%) the most common.

On average survivors reported 2.16 ongoing side effects from their treatment $(S D=1.72)$ with a mean HRQoL of $71.61(S D=21.15)$, most were satisfied with the information they received regarding treatment $(M=6.04 ; S D=1.34)$. The mean Decisional Regret score of 18.02 suggests that levels of regret amongst were reasonably low, yet there was considerable variation here ( $S D=19.52)$. For instance, $28.3 \%$ experienced no regret whatsoever (classified as a score of 0 ), while $14.5 \%$ of survivors experienced high levels of regret (a score above 50).

Fear of recurrence was low on average $(M=29.42$; $S D=20.97)$, yet the majority of survivors (61\%) reported a score of above 25 , and $18.6 \%$ experienced scores over 50.

\section{2 | Multiple regression analysis}

Table 2 displays the results of the regression analysis. Block 1 (sociodemographic characteristics and disease extent) explained $4.5 \%$ of variance in FOR, which was statistically significant $(p<.001)$. Treatment and health-related factors (Block 2) explained an additional $18.0 \%$ of the variance $(p<.001)$, while treatment appraisals (Block 3 ) contributed a further $4.3 \%$ of variance $(p<.001)$. The model as a whole was significant $(p<.001)$ and explained $26.8 \%$ of variance in FOR.

Consistent with our hypotheses, both treatment regret $(\beta=.17$, $p<.001)$ and satisfaction with information received regarding treatment ( $\beta=-.08, p=.003$ ) were significantly associated with FOR after adjusting for sociodemographic, disease, treatment, and health-related factors. Specifically, the lower the survivor's satisfaction surrounding

TABLE 2 Hierarchical regression analyses for variables predicting FOR amongst prostate cancer survivors

\begin{tabular}{|c|c|c|c|c|c|c|c|}
\hline Variables & $\beta$ & $p$ & $t$ & B & SE & \multicolumn{2}{|c|}{$\mathrm{Cl} 95 \%$} \\
\hline \multicolumn{3}{|l|}{ Step 1: sociodemographic characteristics and disease extent } & & & & & \\
\hline Age & -.02 & .57 & -0.57 & -0.06 & 0.11 & -0.27 & 0.15 \\
\hline Education & .01 & .87 & 0.16 & 0.15 & 0.94 & -1.69 & 1.99 \\
\hline Marital status (other = $0 ;$ married/cohabiting $=1$ ) & -.05 & .17 & -1.38 & -2.72 & 1.97 & -6.58 & 1.14 \\
\hline Employment status (other = $0 ;$ employed $=1$ ) & -.05 & 19 & -1.31 & -2.13 & 1.62 & -5.32 & 1.06 \\
\hline Disease classification (localised = 0; locally advanced/advanced = 1) & $.12^{* *}$ & .00 & 3.40 & 6.07 & 1.79 & 2.56 & 9.58 \\
\hline \multicolumn{8}{|l|}{$R^{2}$ Change $=0.05$} \\
\hline \multicolumn{8}{|l|}{ Step 2: treatment and health-related factors } \\
\hline Primary treatment (lower = more invasive) & $.15^{* * *}$ & .00 & 4.00 & 2.19 & 0.55 & 1.11 & 3.26 \\
\hline Current side effects of treatment (higher = more side effects) & $.14^{* * *}$ & .00 & 3.78 & 1.64 & 0.43 & 0.79 & 2.49 \\
\hline GHS (higher = better HRQoL) & $-.29^{* * *}$ & .00 & -7.72 & -0.27 & 0.03 & -0.34 & -0.20 \\
\hline \multicolumn{8}{|l|}{$R^{2}$ Change $=0.18$} \\
\hline \multicolumn{8}{|l|}{ Step 3: appraisals of treatment } \\
\hline Satisfaction with information received (higher = greater satisfaction) & $-.08^{*}$ & .03 & -2.20 & -1.22 & 0.55 & -2.31 & -0.13 \\
\hline Regret over treatment decision (higher = greater regret) & $.17^{* * *}$ & .00 & 4.64 & 0.18 & 0.04 & 0.10 & 0.25 \\
\hline \multicolumn{8}{|l|}{$R^{2}$ Change $=0.04$} \\
\hline $\mathrm{R}^{2}=0.27$ & & & & & & & \\
\hline
\end{tabular}

Statistical significance:

${ }^{*} p<.05$;

${ }^{* *} p<.01$

${ }^{* * *} p<.001$ 
information received and the higher the regret over treatment decision making, the higher the FOR.

A further 4 variables were associated with higher FOR: lower HRQoL $(\beta=-.29, p<.001)$; less invasive treatments $(\beta=.15$, $p<.001)$; advanced disease $(\beta=.12, p<.001)$; and more current side effects $(\beta=.14, p<.001)$.

\section{3 | Sensitivity analysis}

Fear of recurrence did not differ significantly between survivors suspected of having a recurrence and those who were not $(p>.05)$. When the analysis was rerun excluding those suspected of a recurrence, the same 6 variables were significantly correlated with FOR, with similar coefficients as in the original model (see supplementary table). The only difference here was that employment status emerged as an additional correlate of FOR, such that those who were employed at diagnosis had lower FOR ( $\beta=-.09, P=.04$ ).

\section{5 | CONCLUSIONS}

The finding that the majority of PC survivors in our study experienced at least some worry over cancer recurrence, even those who had no suspected evidence of such a recurrence, is in keeping with previous research. ${ }^{7,11}$ Our research goes beyond previous work in that we have shown that men's appraisals of their treatment are significantly associated with FOR, even after adjusting for factors with previously established relationships.

\section{1 | Treatment appraisals and FOR}

Both treatment regret and satisfaction with information received regarding treatment reflect aspects of a survivor's subjective appraisal of their treatment experience. Our findings show how such elements relate to FOR in addition to more objective factors such as cancer stage and health status. This is broadly consistent with the common sense model of FOR, ${ }^{29}$ which holds that FOR is influenced by one's illness representations. It is reasonable to assume that how a survivor views their illness and its treatment forms part of this representation and therefore may impact on FOR. For example, while decisional regret was reasonably low amongst our sample on average, a finding consistent with previous research, ${ }^{18-21}$ its association with FOR illustrates the negative implications, such regret may have on survivor well-being. Indeed, regret over treatment was second only to HRQoL as an FOR correlate, highlighting the significance that treatment decisions may have on later fears and worries.

While little work has focused on the association between decisional regret and FOR, the importance of minimising such regret in health care decisions has been recognised. ${ }^{30,31}$ In PC, research has shown how regret can vary with treatment type, with survivors opting for RP or BT more likely to regret their treatment decisions. ${ }^{19,20}$ Other strong predictors of regret include general health and side effects experienced. ${ }^{18,20}$ In our study, however, regret was significantly associated with FOR even after controlling for these factors, implying that health status and treatment are not sufficient in explaining the relationship between the 2 . This shows that any PC survivor who experiences treatment regret may be at risk of higher levels of FOR, regardless of the treatment(s) they have undertaken or their health status/HRQoL.

We also found that survivors' level of satisfaction with the information received regarding treatment was associated with FOR, pointing to a clear need for health care professionals to provide survivors with sufficient information regarding their treatment options. ${ }^{13}$ The relationship between overall treatment satisfaction and FOR has previously been documented in patients who had undergone RP. ${ }^{32}$ However, this concept of treatment satisfaction could incorporate many factors including survivors' views of the side effects experienced, the time and cost involved in the treatment, as well as their interactions with health care professionals. Our study has isolated a number of key factors that relate to survivors' appraisal of their treatment, which offer some insight into how potentially modifiable factors can influence FOR. Specifically, our results imply that to reduce fears of recurrence, survivors must be happy with the amount of information received regarding treatment and feel comfortable with their decisions.

These findings strengthen the need for health care professionals to support survivors in making fully informed treatment decisions to ensure they are satisfied with information and to guard against future feelings of regret. Given the self-accountable nature of regret, ${ }^{33}$ it is also important that, when making decisions regarding treatment, survivors feel in control. This relates to the notion of health-related self-efficacy, which has been shown to mediate the relationship between symptoms and FOR in PC survivors. ${ }^{12}$ Health-related selfefficacy may be facilitated by decision-making aids. ${ }^{12}$ Such interventions may lead to more positive appraisals regarding treatment thereby reducing uncertainties regarding prognosis and mitigating FOR.

\subsection{Other factors associated with FOR}

As expected, FOR was higher in those diagnosed with more advanced disease, which may reflect a genuinely greater risk of recurrence. This is consistent with previous work, which has shown that both stage and GG have associations with FOR. ${ }^{11,34}$ Interestingly, no sociodemographic factors were associated with FOR. The finding that younger age had no relationship with cancer-related worry contradicts some previous studies. ${ }^{8,34}$ However, a comprehensive review of the FOR literature ${ }^{34}$ suggests that a number of inconsistencies exist regarding the role of demographics in FOR. This suggests that there is no "typical" individual (in sociodemographic characteristics at least) who is more likely to suffer from FOR, implying all patients are potentially at risk at diagnosis.

Our findings do provide evidence for the role of treatment in FOR. Specifically, those survivors who had received more invasive forms of treatment such as RP had lower FOR than those who had less invasive treatment such as ADT. Previous longitudinal analysis showed that survivors undergoing RP had a reduction in FOR after surgery, ${ }^{7}$ which is broadly consistent with our findings; however, no research has compared a range of PC treatments. Our results suggest that those survivors undergoing RP or ERBT may feel that their cancer has been more effectively treated and therefore less likely to recur. Indeed, the perception that more invasive treatments such as surgery can 
reduce the risk of cancer has been documented in other populations. ${ }^{35}$ Conversely, our results suggest that a less interventionist approach to treatment (watchful waiting/active surveillance) may be associated with higher FOR. A possible explanation for this may lie with the uncertainty that abounds for patients undergoing surveillance. ${ }^{36}$ Perhaps, the regular prostate specific antigen testing (and sometimes prostate biopsies) and the knowledge that they are being monitored lead survivors to believe that there is a genuine risk of progression. Given that this is a commonly adopted treatment for PC survivors, especially those deemed low risk, ${ }^{37}$ it is important that health care professionals act to alleviate any concerns such patients may have.

Given the well-established associations between physical health and $\mathrm{FOR},{ }^{38}$ it is not surprising that fears were higher in survivors who reported lower HRQoL. Indeed, the factor most strongly related to FOR was GHS-a finding reflective of extant literature in PC. ${ }^{6}$ Furthermore, we found that FOR was higher in survivors who reported a greater number of ongoing side effects. ${ }^{34}$ This might be because side effects act as an ongoing reminder to survivors of their cancer diagnosis, triggering concerns regarding recurrence. These findings, in conjunction with the fact that side effects are common within PC survivors, even long after treatment has occurred, ${ }^{26}$ further highlight the need to ensure greater use of the range of medications and other supports available to treat side effects.

Consistent with our finding that treatment appraisals impact on FOR, these results demonstrate how survivors' subjective appraisals of their well-being are also important influences. This is again in keeping with the common sense model model of FOR, ${ }^{29}$ which holds that illness representations can include survivors' own appraisals of their physical symptoms, even those unrelated to their cancer diagnosis see also Easterling and Leventhal. ${ }^{39}$

\section{3 | Study limitations}

While this study entailed a comprehensive analysis of possible factors associated with FOR, it does have a number of limitations. We only focused on survivors less than 5-year postdiagnosis, so we cannot say anything about how treatment appraisals might affect FOR in the longer term. The cross-sectional design means we cannot be entirely certain of the direction of the relationship between FOR and treatment appraisals. The presence of a third variable associated with both factors cannot be ruled out-for example, it is possible that depression (eg, that induced by ADT) could cause both high FOR and treatment regret. There are also limitations with some of the measures used. Our measure of satisfaction with treatment information received was only assessed via a single item, while our measure of HRQoL only examined survivors' appraisals of health over the last week rather than since treatment occurred. It is also possible that our measure of recurrence used for the sensitivity analysis did not provide a definite indication of whether recurrence had occurred, given that we did not have access to medical records. Another issue, not unique to this study, is how best to conceptualise FOR given the debate surrounding how to measure this. ${ }^{40}$ Nevertheless, our study controlled for various factors previously shown to be associated with FOR in PC, and despite some missing data, included a sample of PC survivors derived from a population-based sampling frame.

\section{4 | Clinical implications}

The fact that FOR is a reasonably common experience, in spite of generally good prognosis in PC, suggests that those involved in the follow-up of PC survivors must be sensitive to such concerns. Survivors' treatment appraisals, specifically reflections of the treatment experience, are associated with worries over recurrence. These findings suggest that FOR may be mitigated by making survivors fully aware of the options available regarding treatment (and their possible consequences), supporting well-informed treatment decision making, and assisting survivors in their appraisal of treatment and management of any resultant side effects.

\section{REFERENCES}

1. Center MM, Jemal A, Lortet-Tieulent J, et al. International variation in prostate cancer incidence and mortality rates. Eur Urol. 2012 Jun 30;61(6):1079-1092.

2. Bray F, Ren JS, Masuyer E, Ferlay J. Global estimates of cancer prevalence for 27 sites in the adult population in 2008. Int J Cancer. 2013 Mar 1;132(5):1133-1145.

3. Hjertholm P, Fenger-Grøn M, Vestergaard M, et al. Variation in general practice prostate-specific antigen testing and prostate cancer outcomes: an ecological study. Int J Cancer. 2015 Jan 15;136(2):435-442.

4. Siegel RL, Miller KD, Jemal A. Cancer statistics, 2015. CA Cancer J Clin. 2015 Jan 1;65(1):5-29.

5. Watts S, Leydon G, Eyles C, et al. A quantitative analysis of the prevalence of clinical depression and anxiety in patients with prostate cancer undergoing active surveillance. BMJ Open. 2015 May 1;5(5):e006674.

6. Deimling GT, Bowman KF, Sterns S, Wagner LJ, Kahana B. Cancerrelated health worries and psychological distress among older adult, long-term cancer survivors. Psychooncology. 2006 Apr 1;15(4):306-320.

7. Mehta SS, Lubeck DP, Pasta DJ, Litwin MS. Fear of cancer recurrence in patients undergoing definitive treatment for prostate cancer: results from CaPSURE. J Urol. 2003 Nov 30;170(5):1931-1933.

8. van de Wal M, van Oort I, Schouten J, Thewes B, Gielissen M, Prins J. Fear of cancer recurrence in prostate cancer survivors. Acta Oncol. 2016 Mar;1:1-7.

9. Simard S, Savard J, Ivers H. Fear of cancer recurrence: specific profiles and nature of intrusive thoughts. J Cancer Surviv. 2010 Dec 1;4(4):361-371.

10. Bellizzi KM, Latini DM, Cowan JE, DuChane J, Carroll PR. Fear of recurrence, symptom burden, and health-related quality of life in men with prostate cancer. Urology. 2008 Dec 31;72(6):1269-1273.

11. Simard S, Thewes B, Humphris G, et al. Fear of cancer recurrence in adult cancer survivors: a systematic review of quantitative studies. J Cancer Surviv. 2013 Sep 1;7(3):300-322.

12. Torbit LA, Albiani JJ, Crangle CJ, Latini DM, Hart TL. Fear of recurrence: the importance of self-efficacy and satisfaction with care in gay men with prostate cancer. Psychooncology. 2015 Jun 1;24(6):691-698.

13. Aning JJ, Wassersug RJ, Goldenberg SL. Patient preference and the impact of decision-making aids on prostate cancer treatment choices and post-intervention regret. Current Oncology. 2012 Dec;19(Suppl 3): S37

14. Ferrer M, Guedea F, Suárez JF, et al. Quality of life impact of treatments for localized prostate cancer: Cohort study with a 5 year follow-up. Radiother Oncol. 2013 Aug 31;108(2):306-313.

15. Gomella LG, Johannes J, Trabulsi EJ. Current prostate cancer treatments: effect on quality of life. Urology. 2009 May 31;73(5):S28-S35.

16. Fowler FJ, Barry MJ, Lu-Yao G, Wasson J, Roman A, Wennberg J. Effect of radical prostatectomy for prostate cancer on patient quality of life: results from a Medicare survey. Urology. 1995 Jun 30;45(6):1007-1015 
17. Sanda MG, Dunn RL, Michalski J, et al. Quality of life and satisfaction with outcome among prostate-cancer survivors. New England Journal of Medicine. 2008 Mar 20;358(12):1250-1261.

18. Christie DR, Sharpley CF, Bitsika V. Why do patients regret their prostate cancer treatment? A systematic review of regret after treatment for localized prostate cancer. Psychooncology. 2015 Sep 1;24(9):1002-1011.

19. Clark JA, Talcott JA. Confidence and uncertainty long after initial treatment for early prostate cancer: survivors' views of cancer control and the treatment decisions they made. J Clin Oncol. 2006 Sep 20;24(27):4457-4463.

20. Diefenbach MA, Mohamed NE. Regret of treatment decision and its association with disease-specific quality of life following prostate cancer treatment. Cancer Invest. 2007 Jan 1;25(6):449-457.

21. Hu JC, Kwan L, Krupski TL, et al. Determinants of treatment regret in low-income, uninsured men with prostate cancer. Urology. $2008 \mathrm{Dec}$ 31;72(6):1274-1279.

22. Drummond FJ, Kinnear H, O'Leary E, Gavin A, Sharp L. Long-term health-related quality of life of prostate cancer survivors varies by primary treatment. Results from the PiCTure (Prostate Cancer Treatment, your experience) study. J Cancer Surviv. 2015 Jun 1;9(2):361-372.

23. Sharp L, O'Leary E, Kinnear H, Gavin A, Drummond FJ. Cancer-related symptoms predict psychological wellbeing among prostate cancer survivors: results from the PiCTure study. Psychooncology. 2016 Mar 1;25(3):282-291.

24. Greenberg DB, Kornblith AB, Herndon JE, et al. Quality of life for adult leukemia survivors treated on clinical trials of cancer and leukemia group B during the period 1971-1988. Cancer. 1997 Nov 15;80(10):1936-1944.

25. Hill JM, Kornblith $A B$, Jones $D$, et al. A comparative study of the long term psychosocial functioning of childhood acute lymphoblastic leukemia survivors treated by intrathecal methotrexate with or without cranial radiation. Cancer. 1998 Jan 1;82(1):208-218.

26. Gavin AT, Drummond FJ, Donnelly C, O'Leary E, Sharp L, Kinnear HR. Patient-reported 'ever had' and 'current' long-term physical symptoms after prostate cancer treatments. BJU Int. 2015 Sep 1;116(3):397-406.

27. Aaronson NK, Ahmedzai S, Bergman B, et al. The European Organization for Research and Treatment of Cancer QLQ-C30: a quality-of-life instrument for use in international clinical trials in oncology. J Natl Cancer Inst. 1993 Mar 3;85(5):365-376.

28. Brehaut JC, O'Connor AM, Wood TJ, et al. Validation of a decision regret scale. Med Decis Making. 2003 Jul 1;23(4):281-292.

29. Lee-Jones C, Humphris G, Dixon R, Bebbington HM. Fear of cancer recurrence-a literature review and proposed cognitive formulation to explain exacerbation of recurrence fears. Psychooncology. 1997 Jun 1;6(2):95-105.
30. Connolly T, Reb J. Regret in cancer-related decisions. Health Psychol. 2005 Jul;24(4S):S29.

31. Pérez MM, Menear M, Brehaut JC, Légaré F. Extent and Predictors of Decision Regret about Health Care Decisions A Systematic Review. Med Decis Making. 2016 Mar 14. doi: 10.1177/0272989X16636113

32. Hart SL, Latini DM, Cowan JE, Carroll PR. CaPSURE ${ }^{\mathrm{TM}}$ Investigators. Fear of recurrence, treatment satisfaction, and quality of life after radical prostatectomy for prostate cancer. Support Care Cancer. 2008 Feb 1;16(2):161-169.

33. Passyn K, Sujan M. Self-accountability emotions and fear appeals: motivating behavior. J Consum Res. 2006 Mar 1;32(4):583-589.

34. Crist JV, Grunfeld EA. Factors reported to influence fear of recurrence in cancer patients: a systematic review. Psychooncology. 2013 May 1;22(5):978-986.

35. Basu NN, Barr L, Ross GL, Evans DG. Contralateral risk-reducing mastectomy: review of risk factors and risk-reducing strategies. International journal of surgical oncology. 2015 Jan;27:2015

36. Wallace M. Finding more meaning: the antecedents of uncertainty revisited. J Clin Nurs. 2005 Aug 1;14(7):863-868.

37. Loeb S, Berglund A, Stattin P. Population based study of use and determinants of active surveillance and watchful waiting for low and intermediate risk prostate cancer. J Urol. 2013 Nov 30;190(5):1742-1749.

38. Koch L, Jansen L, Brenner H, Arndt V. Fear of recurrence and disease progression in long-term ( $\geq 5$ years) cancer survivors-a systematic review of quantitative studies. Psychooncology. 2013 Jan 1;22(1):1-1.

39. Easterling DV, Leventhal $\mathrm{H}$. Contribution of concrete cognition to emotion: neutral symptoms as elicitors of worry about cancer. Journal of Applied Psychology. 1989 Oct;74(5):787.

40. Lebel S, Simard S, Harris C, et al. Empirical validation of the English version of the fear of cancer recurrence inventory. Qual Life Res. 2016 Feb 1;25(2):311-321.

\section{SUPPORTING INFORMATION}

Additional Supporting Information may be found online in the supporting information tab for this article.

How to cite this article: Maguire R, Hanly P, Drummond FJ, Gavin A, Sharp L. Regret and fear in prostate cancer: The relationship between treatment appraisals and fear of recurrence in prostate cancer survivors. Psycho-Oncology. 2017; 26:1825-1831. https://doi.org/10.1002/pon.4384 